\title{
Estudo retrospectivo de fatores de risco materno, pré e perinatal para paralisia cerebral na rede pública de saúde
}

\author{
Retrospective study of maternal, gestational and labor risk factors for cerebral \\ palsy in the public health system
}

Thamires Máximo Neves Felice ${ }^{1}$, Jair Licio Ferreira Santos² (D), Luzia Iara Pfeifer ${ }^{3}$ (D)

\begin{abstract}
RESUMO
Estudo retrospectivo. Objetivo: Verificar os fatores de risco para paralisia cerebral no período de 5 anos (2010-2014) no município de Ribeirão Preto - SP, buscando identificar a proporção de crianças nascidas na rede pública e que foram identificadas como apresentando risco de atraso de desenvolvimento e, dentre estas, as diagnosticadas com paralisia cerebral, além de identificar os fatores de riscos mais prevalentes e associá-los ao diagnóstico de paralisia cerebral contribuindo, desta forma, para o desenvolvimento de estratégias locais de prevenção e assistência no município. Metodologia: A coleta de dados identificou os bebês de risco e os riscos materno, pré e perinatais nos prontuários das crianças que frequentaram o serviço de referência, onde o desfecho final era o diagnóstico de paralisia cerebral. Foram realizadas análises descritivas e testes de associação. Resultados: Dos nascidos vivos no período, 23,3\% foram considerados com risco de atraso do desenvolvimento. Foram analisados 481 prontuários e em 76,92\% não havia informação da saúde prévia da mãe e a maioria não planejou a gravidez. Cerca de $65 \%$ das crianças apresentavam indícios de alterações neurológicas e 73,4\% tiveram alta por abandono. Conclusão: $O$ estudo identificou as crianças que tiveram risco de atraso e verificou os riscos mais frequentes e o perfil das características pré e perinatais. Devido ao número de altas por abandono, não foi possível verificar o desfecho, comprometendo as análises de associação, estimativa da prevalência de paralisia cerebral e uma intervenção mais direcionada no período de maior neuroplasticidade e melhor prognóstico, sendo necessárias adequações no serviço.
\end{abstract}

Palavras-chave: Paralisia Cerebral; Fatores de Risco; Desenvolvimento Infantil; Epidemiologia.

\begin{abstract}
Retrospective study. Objective: Verifying the risk factors for cerebral palsy in the period of 5 years (2010-2014) in the city of Ribeirão Preto - SP, seeking to identify the proportion of children born in the public system who were identified as presenting risk of developmental delays and, among those diagnosed with cerebral palsy, in addition to identifying the most prevalent risk factors and associating them with cerebral palsy diagnosis, thus contributing to the development of local prevention and care strategies in the County. Methodology: Data collection identified infants at risk and maternal, pre and perinatal risks in the records of children who attended the referral service where the final outcome was the diagnosis of cerebral palsy. Descriptive analyses and association tests were performed. Results: Of the live births in the period, $23.3 \%$ were considered at risk of developmental delay. A total of 481 medical records were analyzed, and in $76.92 \%$ there was no information on the mother's previous health and most of them did not plan the pregnancy. About $65 \%$ of the children had signs of neurological alterations and $73.4 \%$ were discharged from the hospital. Conclusion: The study identified the children who were at risk of delay and verified the most frequent risks and the profile of pre and perinatal characteristics. Due to the number of discharges, it was not possible to verify the outcome, compromising the analysis of association, estimation of cerebral palsy prevalence and a more targeted intervention in the period of major neuroplasticity and better prognosis.
\end{abstract}

Keywords: Cerebral Palsy; Risk Factors; Child Development; Epidemiology.

1. Doutoranda. Faculdade de Medicina de Ribeirão Preto, Universidade de São Paulo (FMRP-USP), Ribeirão Preto (SP), Brasil.

2. Professor titular. FMRP-USP, Ribeirão Preto (SP), Brasil.

3. Professora associada. FMRP-USP, Ribeirão Preto (SP), Brasil.

$\triangle$ Thamires Máximo Neves Felice. Avenida Bandeirantes, 3900 - Laboratório de Ensino e Pesquisa em Terapia Ocupacional, Infância e Adolescência (LEPTOI). CEP: 14051-140. Ribeirão Preto (SP), Brasil. thamiresmnf@gmail.com | Recebido: 04/12/2018 | Aprovado: 09/05/2019 


\section{INTRODUÇÃO}

A paralisia cerebral (PC) descreve um grupo de desordens permanentes relacionadas ao desenvolvimento do movimento e da postura, que causa limitações funcionais atribuídas a distúrbios não progressivos no cérebro fetal ou infantil em desenvolvimento ${ }^{1}$.

Por ser a principal causa de incapacidade física na infância ${ }^{2}$ o acompanhamento deve ser realizado tendo em vista as alterações associadas, como déficits auditivos ou visuais, convulsões, comprometimentos de sensação e percepção, cognitivos, emocionais, comportamentais e musculoesqueléticos $^{1,3}$. Uma meta-análise realizada em 2014 sobre as taxas globais de distúrbios associados e limitações funcionais na PC, registrou que três em cada quatro indivíduos sentem dor; um em cada dois apresenta algum grau de deficiência intelectual; um em cada três não deambula e adquire luxação de quadril; um em cada quatro apresenta problema de linguagem, epilepsia, distúrbio de comportamento, incontinência urinária; um em cada cinco apresenta distúrbio do sono; um em cada dez é deficiente visual ${ }^{3}$.

A PC é geralmente diagnosticada por volta dos 12 a 24 meses devido à falta de marcadores específicos, o que gera uma prática de "esperar para ver"3. O diagnóstico precoce em 12 semanas de idade somente é possível em casos mais graves nas unidades de terapia intensiva neonatal onde os fatores de risco são identificáveis. Por outro lado, diagnosticar precocemente crianças a termo, sem a presença de fatores de risco identificáveis, e com comprometimentos mais leves requer mais pesquisas ${ }^{3}$.

O diagnóstico precoce é considerado a meIhor prática, pois gera a oportunidade de uma rápida intervenção justamente quando são possíveis os maiores ganhos de neuroplasticidade ${ }^{4}$. Segundo Novak (2014) ${ }^{3}$, o diagnóstico da PC envolve a combinação de histórico de fatores de risco, exame neurológico, avaliação da qualidade do movimento, neuroimagem e exclusão de diagnósticos alternativos (como desordens progressivas).

Os fatores de risco são condições ou variáveis ambientais, biológicas, genéticas ou sociais associadas a uma grande probabilidade de ocor- rência de resultados negativos no desenvolvimento infantil em qualquer área 5 .

Evidências recentes apontam para o fato de que a PC pode ser o resultado de uma combinação de fatores que incluem a predisposição genética associada a fatores desencadeantes presentes tanto no ambiente intra como extrauterino ${ }^{3}$. Os fatores de risco mais frequentes em lactentes para paralisia cerebral são: a infecção congênita (15\%), a infecção do sistema nervoso central $(10,6 \%)$ e o estado de mal convulsivo $(22,5 \%)$, sendo que a prematuridade esteve associada a esses fatores de risco em $50 \%$ dos lactentes ${ }^{6}$. Complementarmente a estes dados, a baixa idade gestacional, o baixo peso ao nascer, a asfixia perinatal, a leucomalácia periventricular ou subcortical, a hemorragia intraventricular grave, a isquemia cerebral e a lesão da substância cinzenta profunda podem ser relacionadas como preditores para $\mathrm{PC}^{7}$.

Em um estudo retrospectivo realizado em um hospital de Ribeirão Preto foram analisados, ao acaso, 35 prontuários de crianças com diagnóstico de PC entre 1982 e 1998, com o objetivo de verificar fatores etiológicos. As infecções congênitas (36\%) e agressão hipóxico-isquêmica (28\%) foram os mais frequentes fatores determinantes de PC entre 25 casos com etiologia definida 8 .

Atualmente há 17 milhões de pessoas com PC no mundo e estima-se que em países em desenvolvimento a incidência seja de $7 / 1.000$ nascidos vivos. Há evidências de que as diferenças de prevalências entre países em desenvolvimento e desenvolvidos podem ser atribuídas às más condições de saúde pré-natais e ao cuidado primário deficitário à gestante ${ }^{3}$.

Diante da carência de estudos nacionais sobre a prevalência e incidência da PC e sendo as taxas de extrema importância para a orientação das políticas operacionais de saúde e no auxílio da alocação de recursos adequados para a prevenção, esta pesquisa teve como objetivo verificar os fatores de risco para PC no período de 5 anos (20102014) no município de Ribeirão Preto-SP, buscando identificar a proporção de crianças nascidas na rede pública do município e que foram identificadas como apresentando risco de atraso de desenvolvimento e, dentre estas, as que foram diagnos- 
ticadas com paralisia cerebral, além de identificar os fatores de riscos mais prevalentes e associá-los ao diagnóstico de PC contribuindo, desta forma, para o desenvolvimento de estratégias locais de prevenção e assistência no município.

\section{MATERIAL E MÉTODOS}

O presente estudo é classificado como epidemiológico descritivo, de levantamento retrospectivo. Os critérios de inclusão foram os nascidos vivos na rede pública do município de Ribeirão Preto-SP, no período de janeiro de 2010 a dezembro de 2014, que apresentaram algum risco de desenvolvimento. Todos os bebês de risco nascidos no referido período foram encaminhados ao serviço de Estimulação Precoce, entretanto, nem todos chegaram a este serviço, não sendo possível identificar as causas e, desta forma, não participaram do estudo.

Para identificar estes riscos e coletar os dados, o estudo apresentou duas etapas (Figura 1).

Os prontuários do serviço de Estimulação Precoce não são eletrônicos e ficam armazenados em caixas, as quais estavam em processo de separação e organização. Desta forma, no momento da pesquisa, não havia uma categorização por ano ou por número de cadastro destes prontuários. O procedimento utilizado para a coleta de dados seguiu a lógica de abrir uma caixa, verificar os prontuários elegíveis desta caixa por ano de nascimento, separá-los e, verificar pelo número de cadastro os prontuários que estavam na lista confeccionada por um Programa da Secretaria Municipal de Saúde (SMS) que possui um cadastro geral dos nascidos no Sistema Único de Saúde (SUS) no município.

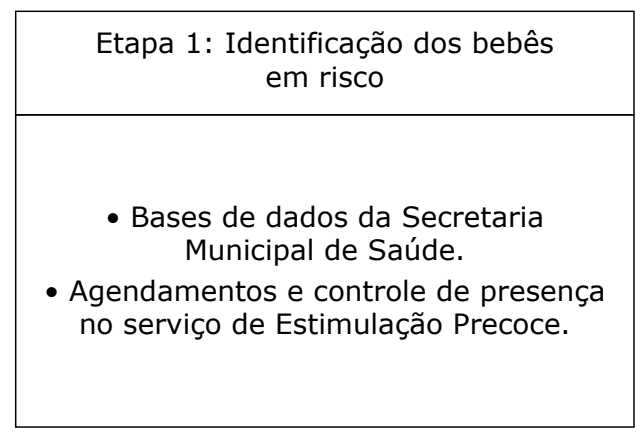

Para identificar o desfecho primário, diagnóstico de paralisia cerebral, foi verificado se no prontuário havia relato do diagnóstico.

As variáveis propostas para a coleta foram categorizadas em fatores de risco materno, fatores de risco gestacional e fatores de risco do parto. Desta forma, foram coletadas informações sobre: endereço, idade da mãe no nascimento da criança, escolaridade da mãe, saúde prévia da mãe, etnia, serviço que realizou o pré-natal, pré-natal adequado, relato de uso de cigarros e/ou drogas e/ou álcool, intercorrências na gestação, idade gestacional, tipo de parto, Apgar no $1^{\circ}$ e $5^{\circ}$ minuto, peso ao nascer, presença de anomalias identificáveis no parto, intercorrências no parto, internações e data do diagnóstico.

Foi realizada uma análise descritiva de todas as variáveis, através do Minitab. Para examinar a significância da associação (contingência) entre duas variáveis foi utilizado o Teste Exato de Fisher, que verifica se a variável da linha e a variável da coluna são independentes, sendo a hipótese nula (HO) que a variável da linha e a variável da coluna são independentes. Em algumas análises foi utilizado o Chi Quadrado de Pearson, tendo em vista condições específicas que levaram a não convergência do teste exato.

Para estimar a força de associação entre as variáveis foi utilizado o Cramérs. Seu valor varia de 0 a 1 , sendo que o valor 0 corresponde a ausência de associação e o valor 1 representa uma associação completa entre as variáveis analisadas.

O projeto de pesquisa foi submetido ao Comitê de Ética em Pesquisa da Faculdade de Medicina de Ribeirão Preto da Universidade de São Paulo e foi aprovado (CAAE: 44249115.3.0000.5440).

Etapa 2: Verificação dos prontuários, coleta dos dados

- Busca ativa de prontuários no serviço de Estimulação Precoce.

- Coleta de dados (aspectos da saúde materna, pré-natal, parto e pós-parto).

Figura 1: Etapas do estudo para coleta das informações. 


\section{RESULTADOS}

Na Etapa 1 foram identificados os bebês de risco de acordo com as bases de dados de um Programa da SMS de Ribeirão Preto através dos encaminhamentos realizados nas maternidades do SUS ou em outros serviços do SUS para acompanhamento de puericultura e pediatria. Os dados são apresentados na Figura 2.

$\mathrm{Na}$ Etapa 2, através da busca ativa dos prontuários, que estavam armazenados em caixas e sem uma categorização por ano ou por número de cadastro no momento da pesquisa, foi possível identificar
481 prontuários, sendo um com óbito, 232 (48,23\%) do sexo masculino, $241(50,10 \%)$ do sexo feminino e oito $(1,67 \%)$ não tiveram o sexo informado.

No prontuário havia informação sobre os dados cadastrais, contendo o endereço de residência da criança. Este endereço foi utilizado para verificar o distrito de saúde no qual a mesma pertencia realizando uma consulta através dos dados da Prefeitura Municipal de Ribeirão Preto (2017c), que divide o município em distritos diferentes, sendo Central 0,83\%, Norte 36,59\%, Sul 2,91\%, Leste 15,80\%, Oeste 39,50\%, Distrito de Bonfim Paulista $0,21 \%$ e não informado $4,16 \%$.

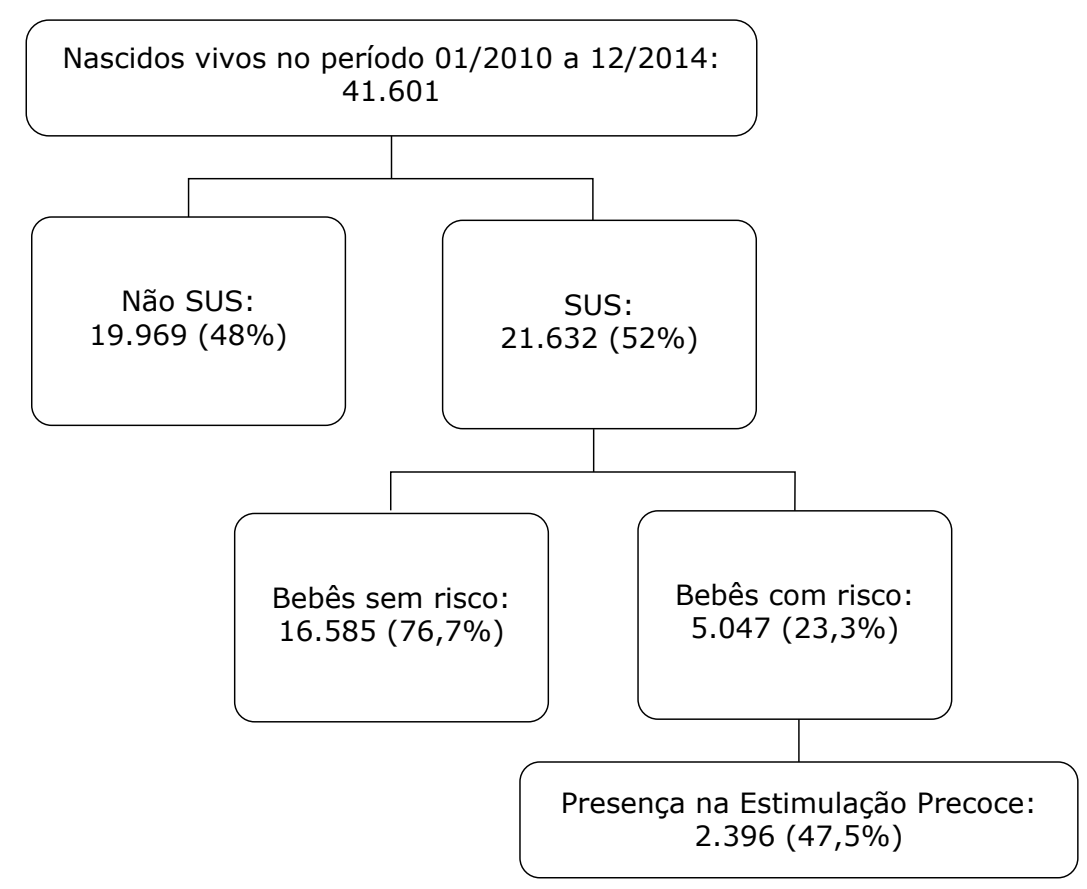

Figura 2: Fluxograma dos bebês de risco identificados pelo Programa da Secretaria Municipal de Saúde e com presença no serviço de Estimulação Precoce.

Na primeira avaliação do bebê de risco havia no prontuário um campo para preenchimento do risco identificado no encaminhamento. Os riscos eram então categorizados no serviço de Estimulação Precoce e na amostra estão representados na Tabela 1, sendo que nos prontuários que havia esta informação $212(47,75 \%)$ possuía um único risco, $154(34,68 \%)$ possuíam dois riscos, $74(16,67 \%)$ possuíam três riscos e quatro $(0,90 \%)$ possuíam quatro riscos.

Com relação aos dados maternos, 97 mães eram casadas $(20,16 \%)$ e em 454 prontuários continha a idade da mãe, variando de 12 a 46 anos, com uma média de 25,9 anos ( $D P \pm 6,99$ ). 
Tabela 1

Frequência e percentual dos riscos dos bebês na amostra.

\begin{tabular}{lcc}
\hline \multirow{2}{*}{ Risco } & Frequência & \multicolumn{2}{c}{ Percentual } \\
& $\mathrm{N}$ & 7,69 \\
Sem informação & 37 & 11,85 \\
Apgar $<5$ no 10 minuto ou $<7$ no 5a minuto & 57 & 7,69 \\
Peso < 2500g & 37 & 7,07 \\
Idade gestacional <37 semanas & 34 & 2,29 \\
Idade gestacional >42 semanas & 11 & 13,72 \\
Ocorrência pós-natal & 66 & 1,46 \\
Anomalias congênitas & 7 & 6,44 \\
Apgar e ocorrência pós-natal & 31 & 11,02 \\
Peso <2500g e IG <37 semanas & 53 & 13,51 \\
Peso <2500g, IG <37 e ocorrência pós-natal & 65 & 5,61 \\
Peso <2500g e ocorrência pós-natal & 27 & 5,61 \\
IG <37 e ocorrência pós-natal & 27 & 6,04 \\
Outras associações & 29 & \\
\hline
\end{tabular}

As informações acerca da saúde prévia da mãe foram coletadas de forma descritiva e agrupadas por categorias, resultando em oito categorias, sendo elas:

a. Aborto: prévio, prévio espontâneo e prévio provocado;

b. Problemas orgânicos: respiratórios (asma e bronquite), hipotireoidismo, diabetes, cardíacos (marca-passo, prolapso) e neurológicos (epilepsia, enxaqueca);

c. Doenças autoimunes: fibromialgia, tumor e lúpus;

d. Problemas psiquiátricos: depressão e síndrome do pânico;

e. Problemas ginecológicos e intercorrências pregressas: (histerectomia, endometriose, mioma, nódulos, ovário policístico, dificuldade para engravidar, útero infantil), doenças sexualmente transmissíveis (sífilis e HIV), intercorrências na gestação e em partos anteriores;

f. Riscos externos: Histórico de uso de drogas, ex-fumante, presidiária;

g. Não informado;

h. Hígida.

As frequência e porcentagem das categorias da saúde prévia da mãe são demonstradas na Tabela 2. Verificou-se que nos 111 prontuários que continha esta informação, apenas três $(0,62 \%)$ eram hígidas, $88(18,30 \%)$ mães apresentavam um único comprometimento de saúde, $13(2,70 \%)$ dois comprometimentos, cinco $(1,04 \%)$ três comprometimentos e duas mães apresentavam quatro e cinco comprometimentos, respectivamente $(0,42 \%)$.

Com relação aos dados gestacionais, em 449 prontuários $(94,13 \%$ dos que haviam relatos) havia a informação de que a mãe realizou o pré-natal. No entanto, não foi possível verificar o número de consultas que a mãe realizou devido ao fato de esta informação não constar em nenhum prontuário.

A gravidez não foi planejada em 56,13\% e não havia informações se foi desejada em $91,06 \%$ dos prontuários. Também não havia informações em $29,31 \%$ sobre uso de drogas, $20,58 \%$ sobre uso de álcool e $19,06 \%$ sobre tabagismo. No entanto, $3,74 \%, 9,36 \%$ e $13,31 \%$ relataram o uso de drogas, álcool e cigarro, respectivamente, na gestação.

Em $338(70,27 \%)$ prontuários havia relatos de intercorrências na gestação e estes relatos foram coletados de forma descritiva e agrupados por categorias, resultando em cinco categorias:

a. Ocorrências clínicas da gestante: infecção de urina, problemas gastrointestinais, 
diabetes, hipertensão, pico hipertensivo, taquicardia, dengue ou suspeita de dengue, sinusite, bronquite, vírus do papiloma humano (HPV) não tratado ou tratado inadequadamente, cólica renal, infecções (toxoplasmose, erisipela, sífilis, hepatite, citomegalovírus), anemia, infecção renal, pielonefrite, candidíase, infecções ginecológicas, paralisia facial de Bell, obesidade, dislipidemia, alteração no triglicérides, hipotensão, tonturas, alimentação não saudável, perda de peso, anorexia, problemas com a tireoide, crise convulsiva;

b. Alterações na gestação: sangramentos, pré-natal de risco (por intercorrências em exames e consultas), placenta envelhecida, descolamento de placenta, hipotonia uterina, necessidade de repouso, risco de aborto, contrações precoces, perda de líquido amniótico, necessidade de inibição do parto, banda amniótica, polihidrâmio, fator $\mathrm{Rh}$ negativo e tomou vacina e teve reação;

c. Pouco preparo: pré-natal tardio, mãe usou medicamentos sem saber que estava grávida, não sabia da gravidez e não fez o pré-natal;

d. Quedas e baques: quedas, queda por acidente automobilístico, deu aula de educação física, trabalhou muito na gestação, sofreu violência do companheiro ou do pai;

e. Problemas emocionais: dificuldade de aceitação, dificuldade para dormir, depressão, relatos de situação e momentos de estresse.

As frequência e porcentagem das categorias das intercorrências na gestação são demonstradas na Tabela 2. Verificou-se que nos 338 prontuários que continha esta informação, 169 (50\%) mães apresentaram uma única intercorrência na gestação, 108 (31,95\%) duas intercorrências, 41 (12,13\%) três intercorrências, 14 (4,14\%) quatro intercorrências e seis $(1,78 \%)$, cinco intercorrências.

Com relação ao parto, $51,14 \%$ foram partos normais, 3,95\% utilizaram fórceps e, em 9,98\% (48 bebês) o parto era múltiplo (gemelar).

A idade gestacional foi preenchida em 470 prontuários, sendo a média de 36,98 semanas (DP $\pm 3,2)$ e variou de 24 a 43 semanas. Na amostra, 199 (42,34\%) era pré-termo (idade ges- tacional menor que 37 semanas), 104 (22,13\%) termo inicial (37 semanas até 38 e 06 dias), $107(22,77 \%)$ termo (39 semanas até 40 e 06 dias), 38 ( $8,09 \%$ ) termo tardio (41 semanas até 41 e 06 dias) e $22(4,68 \%)$ eram pós-termo (42 semanas ou mais).

Já o peso ao nascer foi informado em 471 prontuários e apresentou uma média de $2.692,4$ gramas (DP $\pm 734,9$ ), variando de 560 a 4.885 gramas.

Quando analisado o Apgar, em 432 prontuários havia a informação no primeiro minuto e em 438 no quinto minuto, sendo que em $110(25,46 \%)$ eram abaixo de 5 no primeiro minuto e 19 (4,34\%) eram abaixo de 7 no quinto minuto.

Assim como as intercorrências na gestação, também foram coletadas de forma descritiva as intercorrências no parto, presentes em 66,74\% dos prontuários, e foram agrupadas por categorias, resultando em três categorias:

a. Peri natais: trabalho de parto demorado, pouca e nenhuma dilatação, bolsa rota, placenta grau III, placenta presa, descolamento de placenta, parto induzido, pouco líquido amniótico, polihidrâmio, cesárea de urgência, lesão uterina, problemas com a anestesia para cesárea, presença de infecções, apresentação pélvica e posicionamento não adequado, uso de fórceps, problemas com o cordão umbilical;

b. Com a mãe: alterações na pressão arterial, pico hipertensivo, citomegalovírus, pré-eclâmpsia, infecção, sangramento ou hemorragia no trabalho de parto ou parto, situação estressante, ausência de assistência médica, mãe faleceu no parto, mãe alcoolizada no momento do parto;

3. Com o bebê: anóxia, dificuldade ou desconforto respiratório, hipoglicemia, aspiração ou ingestão de mecônio, icterícia, incompatibilidade sanguínea, sofrimento fetal, sopro cardíaco, cianótico, alterações na estatura do bebê, choro abafado, ausência de movimentos ao nascer, crise convulsiva, parada cardíaca, lavagem do estômago, hipotermia, fratura de clavícula ou costela, precisou amadurecer o pulmão, edemas na cabeça, má formação. 
Tabela 2

Frequência e percentual da saúde prévia da mãe, intercorrências da gestação e do parto.

\begin{tabular}{|c|c|c|}
\hline & $\begin{array}{c}\text { Frequência } \\
\mathrm{N}\end{array}$ & $\begin{array}{c}\text { Percentual } \\
\%\end{array}$ \\
\hline \multicolumn{3}{|l|}{ Saúde prévia da mãe } \\
\hline Sem informação & 370 & 76,92 \\
\hline Aborto & 52 & 10,81 \\
\hline Problemas orgânicos & 18 & 3,74 \\
\hline Doenças autoimunes & 2 & 0,42 \\
\hline Problemas psiquiátricos & 3 & 0,62 \\
\hline Problemas ginecológicos e intercorrências pregressas & 11 & 2,29 \\
\hline Riscos externos & 5 & 1,04 \\
\hline Hígida & 3 & 0,62 \\
\hline Aborto associado a outros fatores & 14 & 2,91 \\
\hline Outras associações & 3 & 0,62 \\
\hline \multicolumn{3}{|l|}{ Intercorrências na gestação } \\
\hline Clínicas da gestante & 178 & 52,82 \\
\hline Alterações na gestação & 31 & 9,20 \\
\hline Pouco preparo & 9 & 2,67 \\
\hline Quedas e baques & 15 & 4,45 \\
\hline Problemas emocionais & 9 & 2,67 \\
\hline Clínicas da gestante e alterações na gestação & 36 & 10,68 \\
\hline Clínicas da gestante e quedas & 17 & 5,04 \\
\hline Clínicas da gestante e problemas emocionais & 10 & 2,97 \\
\hline Outras associações & 32 & 9,5 \\
\hline \multicolumn{3}{|l|}{ Intercorrências no parto } \\
\hline Peri natais & 134 & 41,88 \\
\hline Com a mãe & 30 & 9,38 \\
\hline Com o bebê & 57 & 17,81 \\
\hline Peri natais e com a mãe & 30 & 9,38 \\
\hline Peri natais e com o bebê & 54 & 16,88 \\
\hline Outras associações & 15 & 4,67 \\
\hline
\end{tabular}

As frequência e porcentagem das categorias das intercorrências no parto são demonstradas na Tabela 2.

Verificou-se que nos 321 prontuários que continha esta informação, $157(48,91 \%)$ apresentaram uma única intercorrência no parto, 109 (33,96\%) duas intercorrências, 39 (12,15\%) três intercorrências, $11(3,43 \%)$ quatro intercorrências e cinco $(1,56 \%)$, cinco intercorrências.

O tempo de internação dos bebês no pós-parto variou de 1 a 120 dias, sendo a média 11,15 dias (DP $\pm 16,17)$. Ao ser analisado o local da internação, 355 bebês ficaram internados na enfermaria ou berçário e o tempo variou de 1 a 63 dias, com uma média de 5,82 dias (DP $\pm 7,54$ ). Já nas unidades de terapia intensiva (UTI) ou centros de terapia intensiva (CTI), 127 bebês ficaram internados e o tempo da internação variou de 1 a 120 dias, com uma média de 12,57 (DP $\pm 16,62$ ). Neste local, ainda houve mais 12 relatos de internação, mas sem informação sobre a quantidade de dias.

Para verificar o desfecho, foram anotadas as finalizações dos prontuários e realizadas observações qualitativas.

As finalizações dos prontuários foram separadas em cinco categorias e as observações qualita- 
tivas, que foram coletadas e descritas, também foram separadas posteriormente em três categorias:

\section{a. Bebês com indícios de alteração neu-} rológica: alteração de tônus, presença de clônus, presença de hiperreflexia, relatos de alteração de postura e movimento, relatos de alteração comportamental ou emocional, relatos de desenvolvimento lento ou atraso, exames de imagens com alterações, relatos de crises convulsivas e epilepsia;

b. Bebês sem alterações neurológicas: exames de imagem sem alterações, sem observações relevantes no prontuário;

c. Incompleto: prontuário apenas com a avaliação da criança e relato de pedido de exame de imagem, mas sem o resultado.

\section{Tabela 3}

Frequência e percentual das observações qualitativas e a finalização.

\begin{tabular}{|c|c|c|c|c|c|c|}
\hline Observações / Finalizações & $\begin{array}{c}\text { Alta } \\
\mathrm{n}(\%)\end{array}$ & $\begin{array}{l}\text { Abandono } \\
\mathrm{n}(\%)\end{array}$ & $\begin{array}{c}\text { Encaminhamento } \\
\mathrm{n}(\%)\end{array}$ & $\begin{array}{c}\text { Sem finalização } \\
\mathrm{n}(\%)\end{array}$ & $\begin{array}{l}\text { Óbito } \\
\text { n (\%) }\end{array}$ & $\begin{array}{c}\text { Total } \\
\mathrm{n}\end{array}$ \\
\hline $\begin{array}{l}\text { Indícios de alteração } \\
\text { neurológica }\end{array}$ & $\begin{array}{c}79 \\
(25,24)\end{array}$ & $\begin{array}{c}205 \\
(65,50)\end{array}$ & $\begin{array}{c}15 \\
(4,79)\end{array}$ & $\begin{array}{c}14 \\
(4,47)\end{array}$ & - & 313 \\
\hline $\begin{array}{l}\text { Sem alterações } \\
\text { neurológicas }\end{array}$ & $\begin{array}{c}16 \\
(21,62)\end{array}$ & $\begin{array}{c}58 \\
(78,38)\end{array}$ & - & - & - & 74 \\
\hline Incompleto & - & $\begin{array}{c}52 \\
(96,30)\end{array}$ & $\begin{array}{c}1 \\
(1,85)\end{array}$ & $\begin{array}{c}1 \\
(1,85)\end{array}$ & - & 54 \\
\hline $\begin{array}{l}\text { Indícios de alteração } \\
\text { neurológica e sem } \\
\text { alterações }\end{array}$ & - & $\begin{array}{c}2 \\
(100)\end{array}$ & - & - & - & 2 \\
\hline $\begin{array}{l}\text { Indícios de alteração } \\
\text { neurológica e incompleto }\end{array}$ & - & $\begin{array}{c}34 \\
(94,44)\end{array}$ & $\begin{array}{c}1 \\
(2,78)\end{array}$ & - & $\begin{array}{c}1 \\
(2,78)\end{array}$ & 36 \\
\hline $\begin{array}{l}\text { Sem alterações e } \\
\text { incompleto }\end{array}$ & - & $2(100)$ & - & - & - & 2 \\
\hline Total & $\begin{array}{c}95 \\
(19,75)\end{array}$ & $353(73,39)$ & $\begin{array}{c}17 \\
(3,53)\end{array}$ & $\begin{array}{c}15 \\
(3,12)\end{array}$ & $\begin{array}{c}1 \\
(0,21)\end{array}$ & 481 \\
\hline
\end{tabular}

A Tabela 3 representa o desfecho dos prontuários, com as informações sobre as finalizações e as observações qualitativas coletadas e separadas em categorias.

Muitas variáveis foram analisadas para averiguar possível associação e após análises de evidência $(p>0,05)$, foi verificada associação entre a saúde prévia da mãe e as intercorrências no parto $(p=0,008$ e V Cramér's 0,166$)$.

\section{DISCUSSÃO}

Esta pesquisa teve como objetivo principal realizar um estudo retrospectivo dos fatores de risco para paralisia cerebral no período de 5 anos (2010-2014) no município de Ribeirão Preto-SP, buscando identificar a proporção de crianças nascidas na rede pública do município e que foram identificadas como apresentando risco de atraso de desenvolvimento e, dentre estas, as que foram diagnosticadas com paralisia cerebral, além de identificar os fatores de riscos mais prevalentes e associá-los ao diagnóstico de PC os quais podem ser considerados no desenvolvimento de estratégias de prevenção e assistência no município.

Verificamos que $23,3 \%$ das crianças nascidas no SUS no município apresentaram risco de desenvolvimento, sendo que estes riscos inicialmente foram considerados apenas de saúde ou biológicos notificados pelo Programa da SMS (idade gestacional, baixo peso ao nascer, Apgar, intercorrências pré/peri/pós-parto e anomalias congênitas/genéticas). Há uma carência na literatura sobre os dados incluindo a junção destes riscos, mas quando analisados de formas separadas, um dos fatores de risco que mais nos chama a atenção é a prematuridade.

Até 2012 o Brasil não dispunha de informação nacional sobre a taxa de prematuridade, 
entretanto, através de um inquérito nacional de base hospitalar, realizado em 191 municípios, com 23.894 mulheres entrevistadas, foi revelado que a taxa de prematuridade brasileira é de $11,5 \%$, quase duas vezes superior à observada nos países europeus, sendo que $74 \%$ desses prematuros são considerados como tardios ( 34 a 36 semanas gestacionais) ${ }^{9}$. Em nosso estudo, a taxa de prematuridade no município vai ao encontro da nacional $(11,53 \%)$, contudo, quando analisada a amostra dos prontuários do serviço de Estimulação Precoce, embora a média por nós encontrada tenha sido 36,98 semanas gestacionais, a incidência de prematuridade foi de $42,32 \%$, bastante superior. Sabe-se que a prematuridade, isoladamente ou associada a outro fator, representa um risco para o comprometimento neurológico infantil.

Ainda com relação à prematuridade, alguns estudos $^{10,11,13}$ buscaram identificar os riscos envolvidos no desenvolvimento de desordens neurológicas em prematuros. Ferreira e colaboradores $(2014)^{12}$ constataram que os prematuros apresentam maior risco de desenvolver sepse e, ainda, que crianças com sepse foram 2,5 vezes mais propensas a desenvolver alterações neuromotoras. Nesta mesma direção, Locatelli et al. $(2010)^{10}$, ao analisar 195 casos de prematuridade com idade gestacional menor que 32 semanas constataram que havia desenvolvimento neurológico adverso grave em 45 casos e severo em 28, sendo, destes, 18 casos de PC confirmados. Em concordância, Saldir et al.(2010) ${ }^{11}$ também deram enfoque à prematuridade com igual idade gestacional e verificaram que um em cada quatro recém-nascidos prematuros apresentaram risco no desenvolvimento neurológico.

A prematuridade muitas vezes vem acompanhada do baixo peso ao nascer, e pode ser um dos fatores de risco relevantes para PC em crianças nascidas a termo em países desenvolvidos ${ }^{13}$. Já em bebês a termo, os baixos escores de Apgar podem ser associados a um alto risco para $\mathrm{PC}^{14}$. Estas constatações tornam-se extremamente preocupantes já que nossos dados demonstraram que os baixos escores foram frequentes.

No que diz respeito às intercorrências no período pré-natal e perinatal Serdaroglu et al (2006). ${ }^{15}$ observaram, através de um estudo de prevalência realizado na Turquia, que $26 \%$ das crianças com
PC tiveram danos pré-natais, $18,5 \%$ perinatais e $5,9 \%$ pós-natais. Com este mesmo intuito WU et al. $(2006)^{16}$, demonstraram que $26 \%$ das crianças desenvolveram PC através das seguintes intercorrências: restrição ao aumento intrauterino com peso ao nascimento $<5 \%$ para a idade gestacional $(14 \%)$, má-formação cerebral $(10 \%)$, outras anomalias congênitas (4\%) e infecção congênita por citomegalovírus $(1,6 \%)$. Nos dados adquiridos no presente trabalho encontramos intercorrências em $70,27 \%$ das gestações, sendo as mais frequentes as alterações clínicas da gestante $(52,82 \%)$, o que nos alerta sobre a importância no cuidado materno, principalmente ao verificarmos que em $50 \%$ dos casos apenas uma única intercorrência foi observada e que quando analisadas de forma associada, a mais frequente foi a alteração clínica na gestante com presença de alterações na gestação.

É interessante notar que há indícios de que a prevenção para PC pode estar associada com a melhoria na saúde materna, com o cuidado perinatal e com a prevenção de intercorrências durante a gravidez ${ }^{17}$. Considerando essas informações, chamamos a atenção para o fato de que em $76,92 \%$ dos prontuários não havia informações sobre a saúde prévia da mãe e, nos prontuários em que essa informação existia, era demonstrado, na maior parte das vezes, apenas um comprometimento - sendo o aborto prévio o mais frequente. Mesmo com esta preocupante ausência de dados sobre os aspectos de saúde da mãe, verificou-se que este dado teve associação com as intercorrências no parto.

Outro fato importante a respeito da saúde materna refere-se à assistência pré-natal. Sabe-se que este seguimento quando realizado de forma adequada (com a deteç̧ão e a intervenção precoce das situações de risco, com um sistema ágil de referência hospitalar e com qualificação da assistência ao parto) é um dos grandes determinantes dos indicadores de saúde relacionados à mãe e ao bebê ${ }^{18}$.

Em nosso estudo, $94,13 \%$ das mães foram submetidas a seguimento no período pré-natal, porém, não havia informações sobre a qualidade do mesmo nem sobre número de consultas e, desta forma, torna-se difícil estabelecer a importância destas informações para a ocorrência da PC no município. Nota-se, todavia, que em $56,13 \%$ 
dos casos a gravidez não foi planejada, o que sugere que estes seguimentos podem não ter sido realizados da forma recomendada e, ainda, em $91,06 \%$ dos prontuários não havia informações sobre o desejo e a aceitação da gravidez, o que dificulta novamente nossas reflexões sobre este processo. Apesar desta falta de informações, não podemos ignorar o fato de que a falta de planejamento da gravidez tem uma prevalência preocupante, já que $55,4 \%$ das puérperas de nosso país não planejam sua gravidez, sendo esta média superior aos $40 \%$ da estabelecida para o mundo (cerca de 85 milhões de gestações) ${ }^{9}$.

Outro dado materno interessante foi o de que um em cada cinco prontuários analisados não continha a informação sobre o consumo de drogas, álcool e tabaco. Nos prontuários em que estas informações constavam, 13,31\% das mães relataram que fumaram na gestação. O tabagismo é um fator associado à ocorrência de PC, juntamente com o peso para a idade gestacional ao nascimento, a presença de diabetes mellitus materna, a pré-eclâmpsia, a idade materna maior que 35 anos e 0 fato de a mãe ser primigesta ${ }^{14}$.

A despeito de todos os fatores acima citados, menos de $50 \%$ das crianças nascidas na rede pública do município e identificadas como tendo algum fator de risco compareceram para avaliação no serviço de Estimulação Precoce. Durante nossas buscas não encontramos valores de adesão para uma comparação. Podemos, no entanto, levantar como hipótese que por ser a PC, na maioria das vezes, um diagnóstico tardio e, quando inicialmente as alterações motoras são sutis, os pais e profissionais possivelmente tenham a atitude de "esperar para ver" como já mencionado por Novak ${ }^{3}$. No entanto, estas crianças apresentam um melhor prognóstico se forem atendidas precocemente.

Apesar das lacunas acima citadas é importante discutirmos possíveis hipóteses para a baixa adesão. Em uma primeira análise podemos suspeitar que o hospital em que a criança nasceu pode oferecer também o serviço de acompanhamento do desenvolvimento gerando, desta forma, uma alternativa ao serviço estudado, principalmente com as crianças de maior risco ou com comprometimento motor mais evidentes.
Outra hipótese plausível é a de que o local da residência da criança pode dificultar o acesso até o serviço, sendo que a maioria das crianças da amostra pertence ao distrito oeste e norte, e o serviço de Estimulação Precoce está localizado no distrito leste.

A respeito disso, os distritos oeste e norte compreendem a maioria de nossa amostra e dos usuários do SUS e, os quais são compostos, frequentemente, por famílias com menor índice socioeconômico. Um estudo recente em Gaborone, Botswana ${ }^{19}$ tentou compreender os fatores de risco únicos que afetam as crianças com PC que moram em local onde há poucos recursos. Os pesquisadores, através de um estudo caso-controle, observaram 56 indivíduos e verificaram que os fatores de risco significativos para a PC incluíram história de infecção neonatal grave, complicações durante o parto e vírus da imunodeficiência humana materna, e alertam para os fatores de riscos únicos que podem ser prevenidos.

Em nosso estudo, quase metade das crianças apresentava um único fator de risco, sendo encaminhadas para o serviço de Estimulação Precoce devido principalmente a presença de ocorrência pós-natal. Isto também se repetiu quando analisadas as intercorrências na gestação e no parto, e nos chama a atenção pelo fato de sabermos que, quanto maior o número de fatores de risco presentes, maior a gravidade do impacto no desenvolvimento infantil e que as crianças de países de baixa e média renda estão expostas a um maior número de riscos ${ }^{20}$.

Ainda no que diz respeito às populações de baixo recurso, Bearden et al. (2016) ${ }^{21}$ através de entrevistas com cuidadores em Botswana, revisão de prontuários e exame físico, verificaram a existência de 68 crianças com PC, sendo $41 \%$ do sexo masculino. As etiologias mais comuns para a ocorrência de PC foram os eventos hipóxicos intraparto (18\%), infecções pós-natais (15\%), prematuridade $(15 \%)$, acidentes vasculares cerebrais isquêmicos focais (10\%) e infecções pré-natais $(10 \%)$. A deficiência motora grave foi comum, com a categoria mais grave presente em $41 \%$ e, as comorbidades predominantes foram comprometimento cognitivo (84\%), epilepsia $(77 \%)$ e deficiência visual (46\%). 
Um estudo caso-controle ${ }^{14}$ buscou identificar fatores perinatais associados a PC baseado em registros que incluíam 2.303 crianças nascidas na Suécia (1984-1998) com diagnóstico de PC e 1,6 milhões de lactentes sem o diagnóstico de PC. Foi apurado que os bebês nascidos prematuros tiveram um risco aumentado de PC e constituíam $35 \%$ de todos os casos e, por fim, que os meninos tiveram um maior risco para o desenvolvimento desta condição.

Outro ponto crítico encontrado em nossas análises diz respeito ao diagnóstico clínico. Nossos dados demonstram que $65,07 \%$ das crianças tinham indícios de alterações neurológicas, entretanto, em nenhum prontuário constava o diagnóstico de PC. Além disso, em 73,39\% dos prontuários havia como finalização o abandono do seguimento, o que torna o desfecho mais uma vez inconclusivo.

Sem a possibilidade de um desfecho final e, com apenas relatos de possíveis alterações neurológicas, a situação agrava-se ainda mais pela ausência de um check list e de uma avaliação padronizada, tornando as observações extremamente subjetivas e favorecendo o uso de terminologias não adequadas - quando não apenas especulações.

Para o planejamento de uma adequada intervenção seria necessária uma avaliação criteriosa que excedesse a impressão clínica. A respeito disso, para a identificação precoce de atrasos no desenvolvimento infantil existem diferentes testes de triagem descritos na literatura que possibilitam o encaminhamento para diagnóstico e intervenção precoces ${ }^{22}$, bem como os critérios considerando a combinação de histórico de fatores de risco, exame neurológico e a utilização do HINE, avaliação da qualidade do movimento, neuroimagem e exclusão de diagnósticos alternativos $^{3}$. Ademais, o período em que a intervenção é proposta deve ser considerado, já que nos primeiros anos de vida existe uma maior plasticidade cerebral que possibilita a otimização de ganhos no desenvolvimento de crianças que receberam a estimulação precoce adequada ${ }^{23}$.

Embora não tenha sido possível analisar as associações dos fatores de risco com a PC no município, foram realizadas análises para tentar entender melhor os possíveis motivos do abandono.
Verificamos que o tipo de finalização teve associação com a intercorrência no parto, com os partos múltiplos e com o Apgar $<7$ no quinto minuto. Os partos múltiplos, presentes em cerca de $10 \%$ dos nossos casos, também tiveram associação com as observações qualitativas.

O grande número de altas por abandono também é digno de nota. Além de dificultar as conclusões sobre o desfecho da paralisia cerebral, esta variável nos faz refletir sobre a efetividade do acompanhamento com retornos espaçados realizados bimestralmente ou trimestralmente, não sendo possível a criação de uma rotina e, consequentemente, uma melhor adesão ao acompanhamento.

A identificação de relações causais na PC tem sido desafiadora, e um fator contribuinte para isto pode ser a falta de informações armazenadas em meio digital. O serviço atualmente possui o prontuário eletrônico, porém, as informações continuam sendo armazenadas em papel. As informações armazenadas em meio digital e com uma sistematização na coleta possivelmente facilitariam as análises e tornariam o controle de informações mais ágil e eficaz. Nos Estados Unidos, Europa e Austrália há sistemas de informação que realizam revisões de registros e se baseiam em dados da população advindos de múltiplas fontes comunitárias para o diagnóstico e tratamento de crianças com deficiências de desenvolvimento ${ }^{24}$.

Por ser um estudo retrospectivo, a perda dos dados é um fator de enfraquecimento metodológico e, esta fraqueza pode nos levar a uma subestimação acerca da importância dos fatores de risco e, até mesmo a incidência da condição em questão na população estudada ${ }^{25}$, fatos estes notados claramente em nossas coletas e análises.

\section{CONCLUSÃO}

Este estudo buscou identificar os fatores de riscos mais frequentes para paralisia cerebral e o perfil das características pré-natais e perinatais nas crianças nascidas na rede pública do município de Ribeirão Preto no período de 2010 a 2014. Devido, no entanto, ao alto número de altas por abandono deste serviço de Estimulação Precoce, não foi possível verificar o desfecho do acompanhamento, bem como saber se a criança evoluiu 
para o diagnóstico de $\mathrm{PC}$, já que o diagnóstico não constava em nenhum prontuário avaliado.

A proporção de crianças identificadas como apresentando risco de atraso de desenvolvimento foi de $23,3 \%$ de todos os nascidos vivos no município de Ribeirão Preto. Não foi possível, porém, fazer uma comparação com os dados do Ministério da Saúde, já que o referido órgão subgrupa as crianças em riscos específicos e não globais. Considerando somente a prematuridade de forma isolada, o município possui a mesma proporção nacional (acima da recomendada).

Consideram-se como limitações do estudo o seu modelo retrospectivo e, ainda, o fato dos registros (já existentes em prontuários) serem preenchidos por diferentes profissionais. Estas ausências de dados comprometem, portanto, as análises de associação e a estimativa da prevalência de PC na rede pública no município de Ribeirão Preto, sendo que outras estratégias mais tardias de identificação de risco também devam ser consideradas. Sugere-se uma maior atenção aos riscos maternos prévios, um melhor planejamento da gestação, uma reestruturação do serviço analisado tendo em vista o alto número de abandono do seguimento e uma adequação na detecção de riscos com avaliações padronizadas, com a criação de um check list, além de um sistema de informação que seja capaz de criar um banco de dados de registros para acompanhamento e notificação.

\section{REFERÊNCIAS}

1. Rosenbaum P, Paneth N, Leviton A, Goldstein M, Bax M, Damiano $D$, et al. A report: the definition and classification of cerebral palsy April 2006. Dev Med Child Neurol Suppl 2007;109:8-14.

2. Morris C. Definition and classification of cerebral palsy: a historical perspective. Dev Med Child Neurol Suppl. v2007;109:3-7.

3. Novak I. Evidence-based diagnosis, health care, and rehabilitation for children with cerebral palsy. J Child Neurol 2014; 29(8):1141-56.

4. McIntyre S, Morgan C, Walker K, Novak I. Cerebral palsy-don't delay. Dev Disabil Res Rev. 2011;17(2):114-29.

5. Reppold CT, Pacheco J, Bardagi M, Hutz C. Situações de risco e vulnerabilidade na infância e na adolescência: aspectos teóricos e estratégias de intervenção. Casa do Psicólogo. 2002;7-51.
6. Tâmega IDE, Filho ADAB, Pinto EALDC. Growth in children with encephalopathy, a longitudinal study from the 6th to the 24th month. Int J Nutr Metab. 2011;3(6):65-71.

7. Gladstone M. A review of the incidence and prevalence, types and aetiology of childhood cerebral palsy in resource-poor settings. Ann Trop Paediatr. 2010;30(3):181-96.

8. Funayama CAR, Penna MA, Turcato MF, Caldas CAT, Santos JS, Moretto D. Paralisia cerebral diagnóstico etiológico. Medicina. 2000;33:155-60.

9. Leal MD, Esteves-Pereira AP, Nakamura-Pereira M, Torres JA, Theme-Filha $M$, Domingues RM, et al. Prevalence and risk factors related to preterm birth in Brazil. Reprod Health. 2016;13(3):127.

10. Locatelli A, Andreani M, Pizzardi A, Paterlini G, Stoppa P, Ghidini A. Antenatal variables associated with severe adverse neurodevelopmental outcome among neonates born at less than 32 weeks. Eur J Obstet Gynecol Reprod Biol. 2010;152(2):143-7.

11. Saldir M, Sarici SU, Bakar EE, Ozcan O. Neurodevelopmental status of preterm newborns at infancy, born at a tertiary care center in Turkey. Am J Perinatol. $2010 ; 27(2): 121-8$.

12. Ferreira RC, Mello RR, Silva KS. Neonatal sepsis as a risk factor for neurodevelopmental changes in preterm infants with very low birth weight. J Pediatr (Rio J). 2014;90(3):293-9.

13. McIntyre S, Taitz D, Keogh J, Goldsmith S, Badawi N, Blair E. A systematic review of risk factors for cerebral palsy in children born at term in developed countries. Dev Med Child Neurol. 2013;55(6):499-508.

14. Thorngren-Jerneck K, Herbst A. Perinatal factors associated with cerebral palsy in children born in Sweden. Obstet Gynecol. 2006;108(6):1499-505.

15. Serdaroğlu A, Cansu A, Ozkan S, Tezcan S. Prevalence of cerebral palsy in Turkish children between the ages of 2 and 16 years. Dev Med Child Neurol. 2006;48(6):413-6.

16. Wu YW, Croen LA, Shah SJ, Newman TB, Najjar DV. Cerebral palsy in a term population: risk factors and neuroimaging findings. Pediatrics 2006; 118(2):690-7.

17. Westbom L, Hagglund G, Nordmark E. Cerebral palsy in a total population of 4-11 year olds in southern Sweden. Prevalence and distribution according to different CP classification systems. BMC Pediatr. 2007;7:41.

18. Brasil. Atenção ao pré-natal de baixo risco. Ministério da Saúde. 2012.

19. Monokwane B, Johnson A, Gambrah-Sampaney C, Khurana E, Baier J, Baranov E, et al. Risk Factors for Cerebral Palsy in Children in Botswana. Pediatr Neurol. 2017;77:73-7.

20. Singla DR, Kumbakumba $E$, Aboud FE. Effects of a parenting intervention to address maternal psychological wellbeing and child development and growth in rural Uganda: a community-based, cluster randomised trial. Lancet Glob Health. 2015;3(8):458-69.

21. Bearden DR, Monokwane B, Khurana E, Baier J, Baranov E, Westmoreland $\mathrm{K}$, et al. Pediatric Cerebral Palsy in 
Botswana: Etiology, Outcomes, and Comorbidities. Pediatr Neurol. 2016;59:23-9.

22. Rydz D, Shevell MI, Majnemer A, Oskoui M. Developmental screening. J Child Neurol. 2005; 20(1):4-21.

23. Formiga CK, Pedrazzani ES, Tudella E. Desenvolvimento motor de lactentes pré-termo participantes de um pro- grama de intervenção fisioterapêutica precoce. Rev Bras Fisioter. 2004;8:239-45.

24. Pakula AT, Van Naarden Braun K, Yeargin-Allsopp M. Cerebral palsy: classification and epidemiology. Phys Med Rehabil Clin N Am. 2009;20(3):425-52.

25. Anthonisen NR. Retrospective studies. Can Respir J. 2009;16(4):117-8. 